\title{
The astronomical observatory of the University of Havana. A project for its rehabilitation as a center for science popularisation
}

\author{
Mónica de la Guardia Durán \\ Departamento de Cultura Cientílfica "Félix Varela", \\ Facutad de Física, Universidad de La Habana \\ Colina Universitaria, Vedadao, La Habana, Cuba \\ email: monica@fisica.uh.cu
}

\begin{abstract}
The project aims to recover the Astronomical Observatory of the University of Havana (AOUH) for science outreach at the national level, in order to help increasing the interest in science primarily among children and youth.
\end{abstract}

Keywords. Scientific culture, science outreach, observatories, science and technology museums

\section{Introduction}

In Cuba, as elsewhere in the world, there is a need to increase the scientific knowledge of people as part of their general culture. Such a need grows as we move forward towards a society which development is increasingly based on knowledge (Fensham 2008; Cheng \& Leung 1989).

A major concern is stimulating the interest of students from different academic levels to learn natural and exact sciences. We want our children and youth to feel attracted by science, yet, have we stopped to think how to make science attractive? Most of the time the answer is there, very close to us, and we do not notice it. We do not even realise about all the potential we can make very good use of, in our very own institutions, by using spaces we have let to die in oblivion.

In view of such needs, the Scientific Culture Department "Félix Varela" of the University of Havana, with the leading of Dr. Edwin Pedrero, has considered, since mid-2008, the pertinence and the possibility of promoting steps to recover the Astronomical Observatory of the University of Havana (currently set aside as an observatory and destined to other purposes), aimed at turning it in the near future into a centre for spreading knowledge about astronomy and other related sciences.

\section{A short history of the observatory}

The idea of building a small astronomical observatory at the university was established at the beginning of 20th century. The date of the observatory construction plans is 1914, and the work was assigned to the Public Works Department. At that time, the University of Havana was known as National University, and the Observatory would report to the Department of Cosmography, Astronomy and Complements of Astronomy.

We know that the Department operated, at least, until the 1960s and multiple investigations were performed, tutored by professors Dr. Manuel Rabiña, Dr. Roberto Peña and Dr. Aurelio Martínez, among others (e.g. Pita y Romero 1960). 


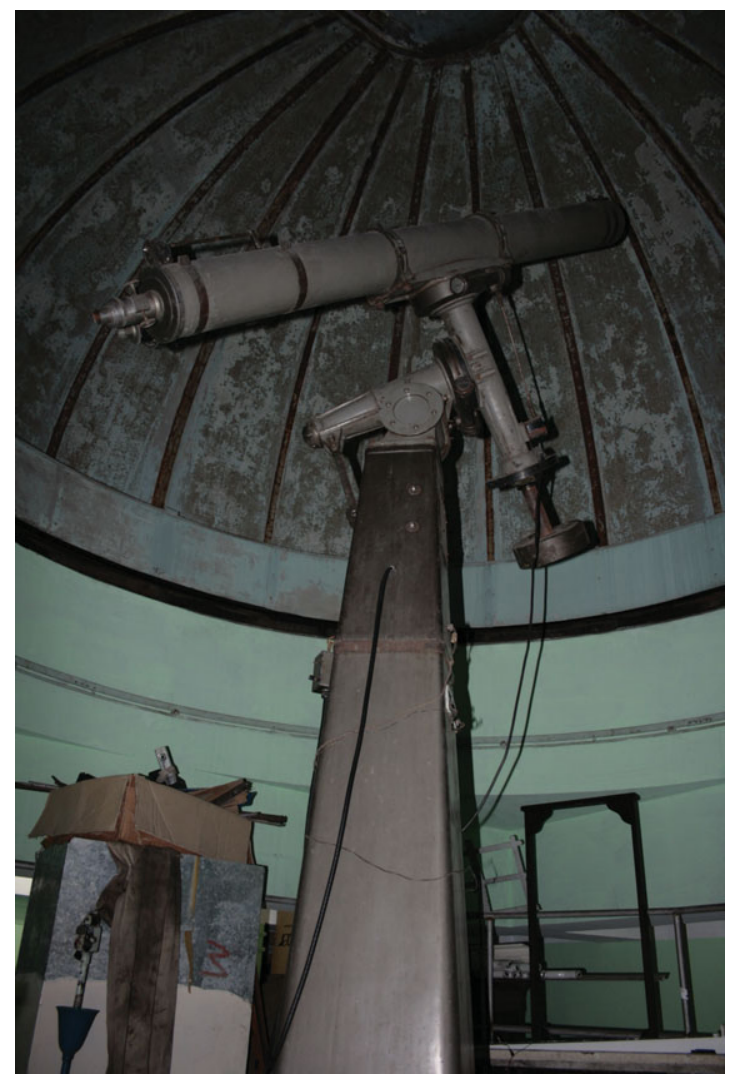

Figure 1. The main $150 \mathrm{~mm}$ equatorial telescope.

At one time, these studies were suspended and the C Department disappeared, so the observatory was gradually losing its function, until it ended abandoned.

In the 1990s, some amateurs and students from the Faculty of Physics made sporadic observations in the observatory of important events, such as the passage of Comet Hyakutake in 1996. From 1998 until 2004, the place hosted the Havana City amateur group "Cosmos". During this time, many observations were made primarily with the main telescope. In 2004, "Cosmos" stopped using the observatory, and it was closed and unused. Two years ago, the Faculty of Physics began using it as storage, and for practical classes of electronics, function that it currently holds.

\section{The treasures of OAUH}

The project constitutes a necessity for the development of the University of Havana, since it is conceived to facilitate the recovery of a space within the university campus that is quite dilapidated, regarding as much the building as the instruments and didactic material it contains.

Most of these instruments and materials have patrimonial, museological, didactic and scientific-technical value. Thus, with some time and monetary resources, they can be usable in the training of professionals in the branches of physics, mathematics and chemistry; as well as in the formation of a scientific vocation in children and adolescents. 


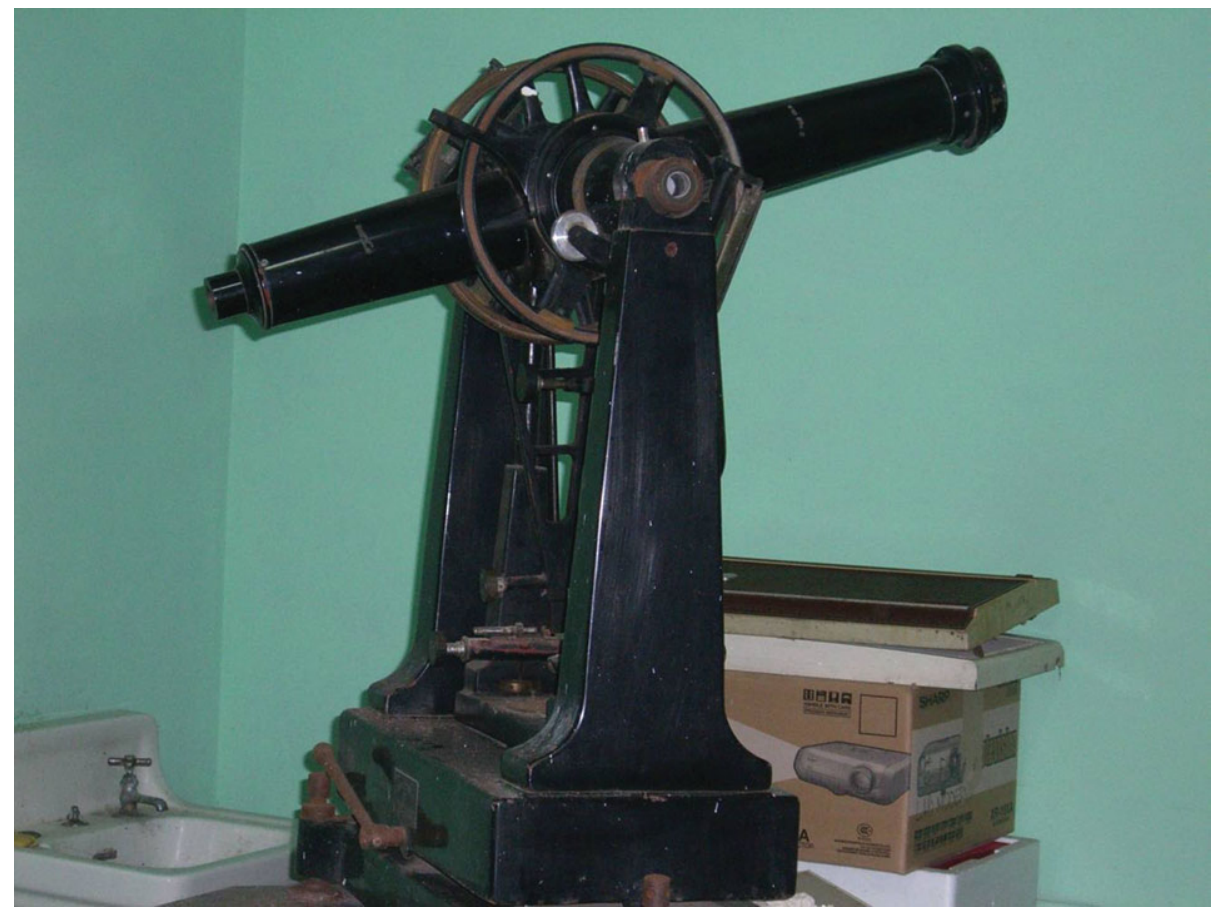

Figure 2. The Meridian Circle.

Astronomy turns out to be a science to which the general public is very much attracted, and it can become the first step into the fascinating world of sciences.

The main instruments in the Observatory include the following:

(a) Main Telescope: Equatorial refractor, 150-mm (Fig. 1). Manufactured in 1949 by Perkin-Elmer Corporation. Objetive and eyepieces missing.

(b) Meridian Circle (Fig. 2). It was the largest of its time in Cuba, and today it is probably the only one. Gaertmer Scientific Corporation. Eyepieces missing.

(c) Secrétan telescope: Equatorial refractor, 95-mm (Fig. 3) The second largest in the observatory. Manufactured in 1861 by Secretan. Eyepieces and finder missing.

The AOUH also treasures other didactical material having patrimonial value (see Figs. 4,5,6), which will need careful restoration: Celestial spheres; models of planetary systems; glass-plate transparencies displaying paintings, illustrations and photographs, brought from Paris and dating back to the 19th century; books, yearbooks, catalogues, some of them of the 19th century.

In addition, it keeps other astronomical and meteorological instruments (sidereal clock, thermometer, thermograph, etc.) proper of the conception of astronomical observatories of the time.

\section{The project}

Our project is still in its first steps. It has already had the approval of the Rector of the University of Havana, and the support of the Dean of the Faculty of Physics.

Among the main institutions involved in the project are:

- Faculty of Physics, leading the project;

- The Institute of Material Research and Engineering of the University of Havana, 


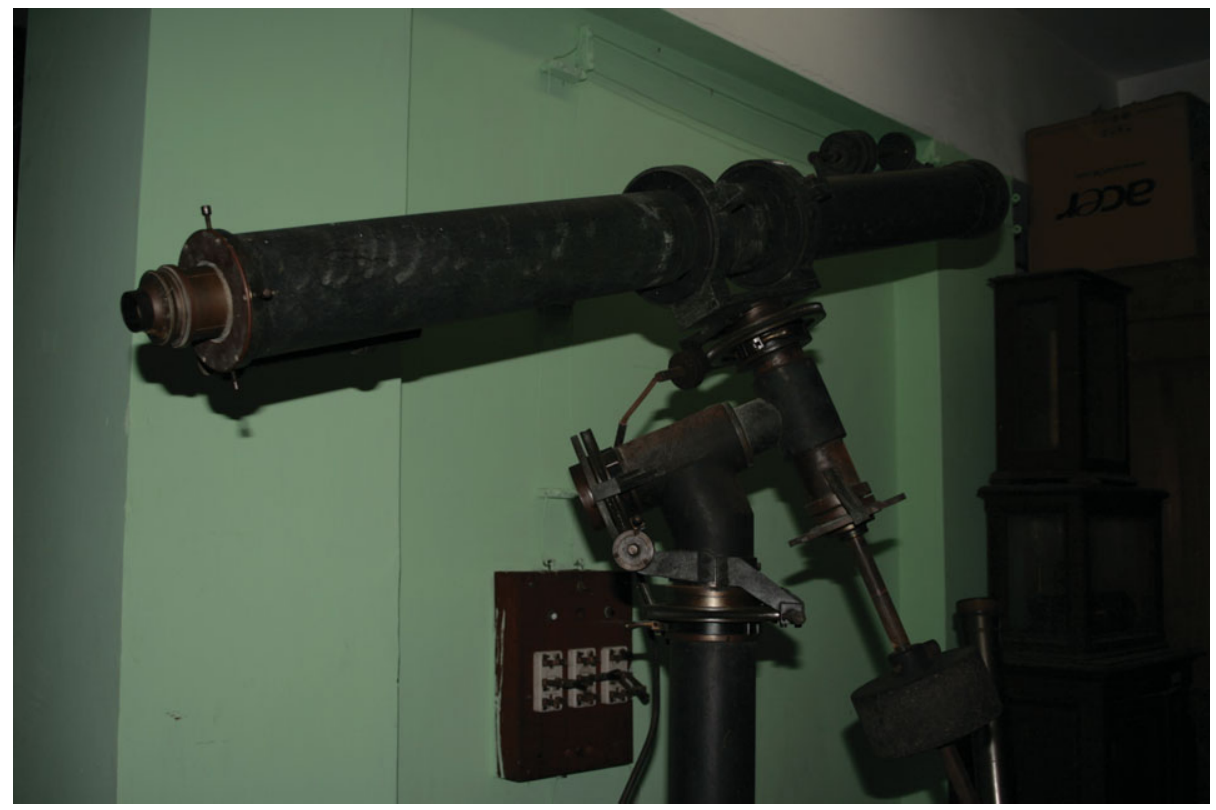

Figure 3. The $95 \mathrm{~mm}$ Secrétan telescope.

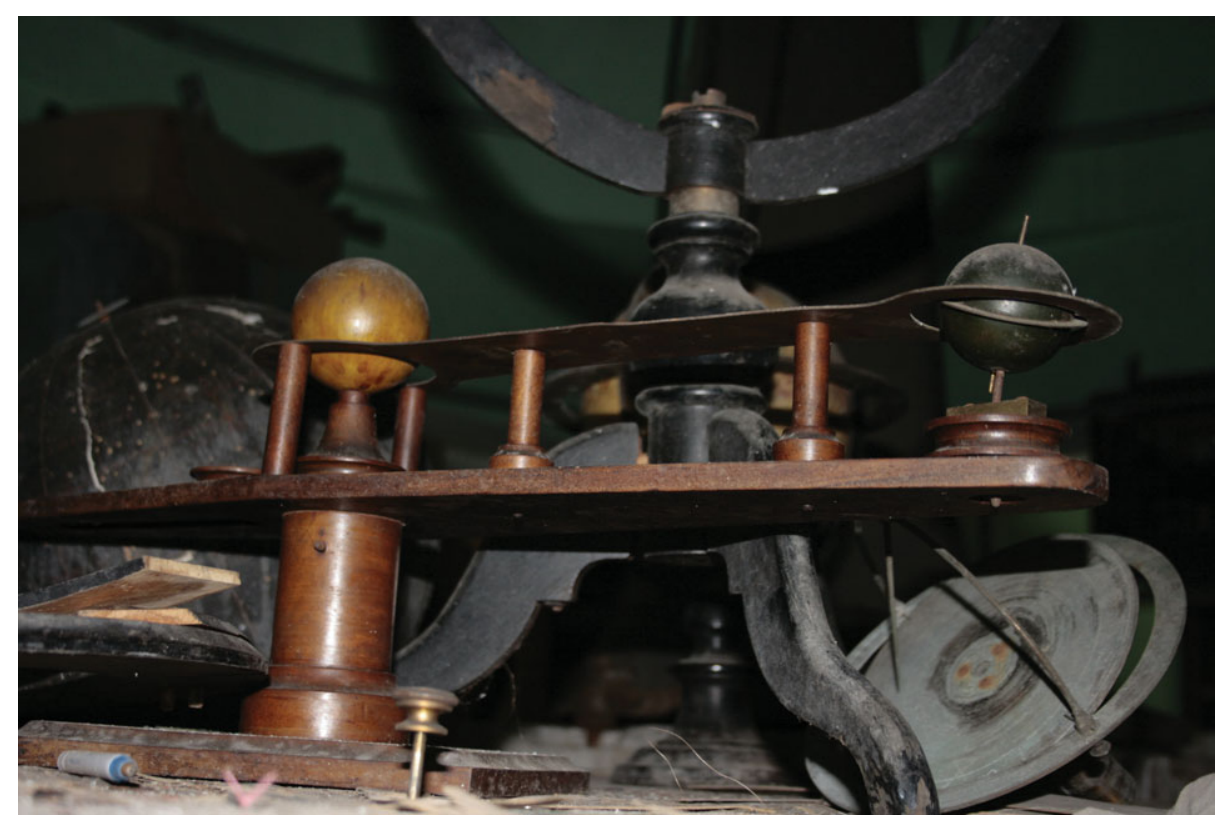

Figure 4. Secrétan scales.

with whom we have been doing some work on diagnosis of the AOUH technical and constructive situation;

- The Geophysics and Astronomy Institute, the only institution of its kind in the country, whose participation in the project is essential to carry out all the work, and 


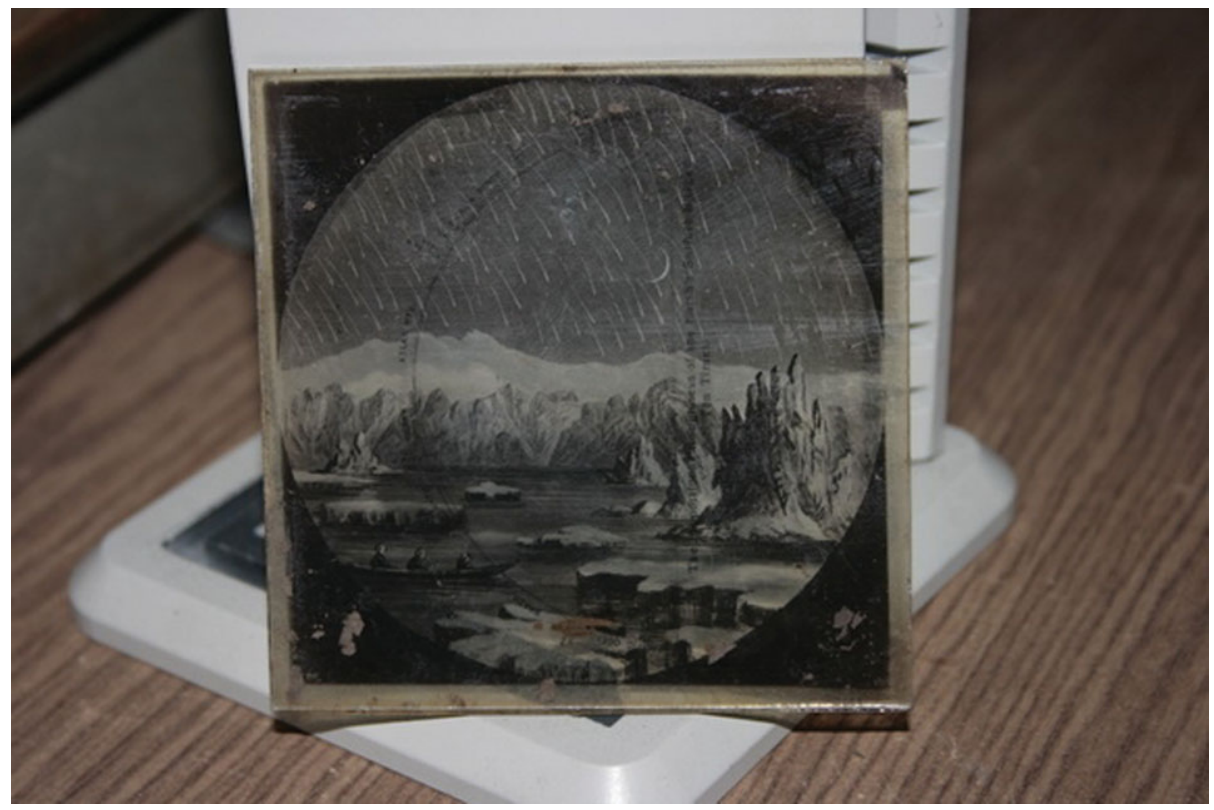

Figure 5. Illustrated glass-plate transparencies.

who has been working side by side for the diagnosis of the technical instruments of the observatory, among others;

- Faculty of Mathematics and Computing, the physical placement of the observatory;

- Faculty of Restoration and Museology, with whom we work closely to perform the restoration and conservation of the materials available to the AOUH;

- Sciences Education Department, of the Ministry of Education;

- Sciences Department of the Ministry of Science, Technology and Environment.

The project's schedule starts with the reconstruction and repair of the civil infrastructure and the equipment needed for the functioning of the AOUH (especially the dome, the opening of the meridian circle, the main telescope, and the meridian circle), which are currently in a state of neglect.

We give a great deal of importance to finding out more about the history of the AOUH, its date of creation, the rôle it played in the university during the years in which it was fully operative, and so forth. Depending on the information we will obtain, we have the initial goal of including the Observatory in the list of heritage of the University of Havana.

At the same time, we have plans to work actively, within the context of IYA 2009, on making astronomy knowledge available to the general public, which will serve as a starting point in our quest to use the observatory to spread scientific knowledge in the future. We can perform actions that do not require the whole of the observatory equipment to be operative. These include public activities, participation in national initiatives in the context of IYA 2009, promotion of the event in the mass media, etc. We intend to somehow bring the AOUH to life, even with the limitations posed by its current condition.

Among the main activities that could make the AOUH in the future are: amateur astronomical observations; astronomy workshops with students of differentacademic 


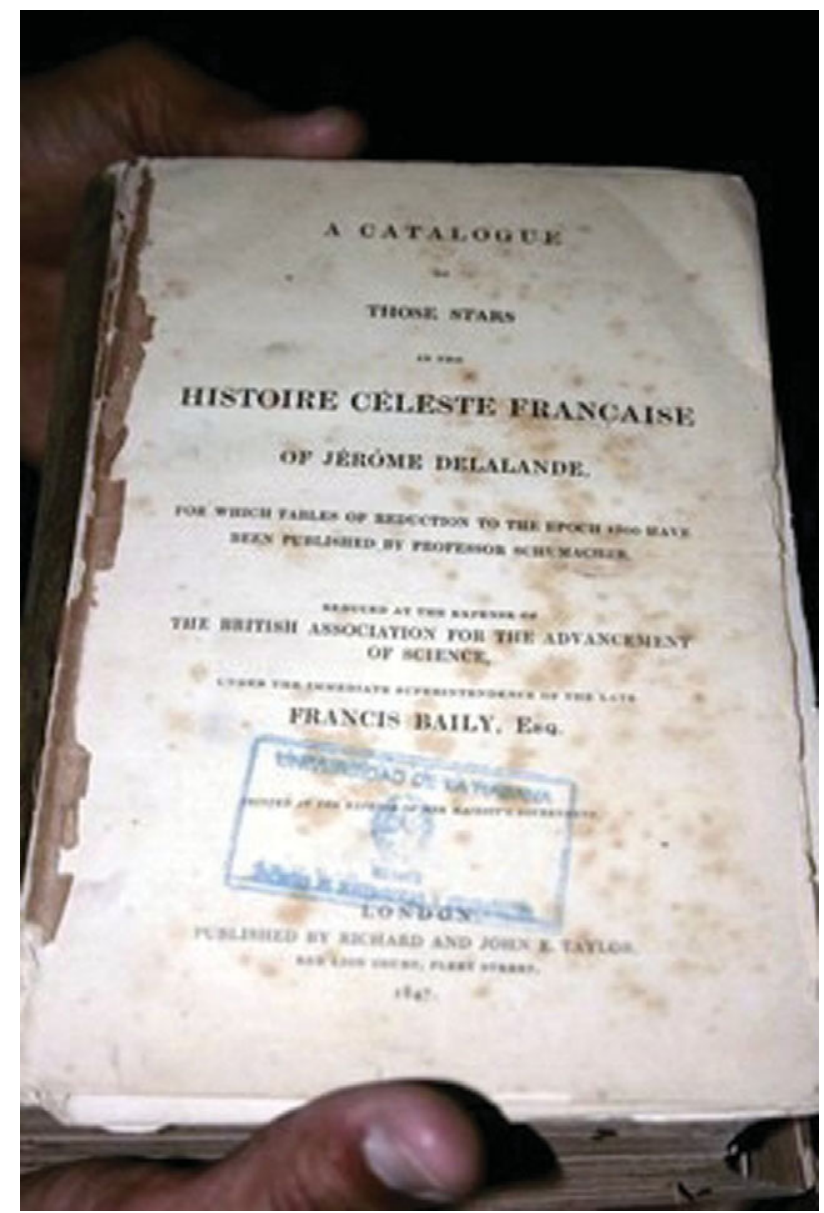

Figure 6. Stellar catalogue of the Histoire Céleste française by Jerôme Delalande, 1847 English edition.

levels; broadcast news and outreach materials in the media; directed visits, organisation of documentaries projection and talks; observation and outreach activities with comunity schools; training of teachers of primary, secondary and pre-university academic levels in matters of astronomy and the use of a telescope.

The greatest aspiration of the project is, once the Observatory has been repaired, developing an automated system that will allow us to perform astronomical observations by remote access, which shall be available from any computer in the country, connected to the Education System National Network.

Currently we have no funding, and the University of Havana has no funds that can be used for these purposes, hence the search for international cooperation with other institutions is of vital importance to us. So far, everything that has been projected has been possible thanks to the efforts of several people from the institutions mentioned before, who have dedicated part of their time to work on it, and call attention to the observatory. Beginning in September, and as a result of these efforts, the Faculty of Physics will have a dedicated person to work at the AOUH, and may be carried out serious work with the inventory of the observatory, to investigate more deeply into its history and to concrete outreach actions. 


\section{References}

Cheng, K. M. \& Leung, K. F. 1989, Popularization of science and technology. What Informal and Nonformal Education Can Do? (Paris: UNESCO) $\dagger$

Pita y Romero, M. 1960, Selección de estrellas para determinar la latitud de La Habana utilizando el método de Talcott. Escuela de Ciencias de la Universidad de La Habana. Tesis de grado para optar al titulo de Dr. en Ciencias Físico-Matemáticas. La Habana, 1960.

Fensham, P. J. 2008, Science education policy-making: eleven emerging issues (Paris: UNESCO) $\ddagger$

$\dagger$ available at : unesdoc.unesco.org/images/0012/001263/126341e.pdf

$\ddagger$ available at : unesdoc.unesco.org/images/0015/001567/156700e.pdf 